\title{
Right atrial to left atrial area ratio on early echocardiography predicts long-term survival after acute pulmonary embolism
}

\author{
Vincent Chow ${ }^{1}$, Austin Chin Chwan Ng${ }^{1}$, Tommy Chung ${ }^{1}$, Liza Thomas ${ }^{2}$ and Leonard Kritharides ${ }^{\text {* }}$
}

\begin{abstract}
Background: Current guidelines recommend that transthoracic echocardiography (TTE) should be performed for acute risk stratification following acute pulmonary embolism (PE), but it is unclear whether the initial TTE can predict long-term outcome beyond six months. We sought to assess the potential of the initial right atrial (RA) to left atrial (LA) area ratio (RA/LA ratio) on TTE to predict long-term mortality in survivors of submassive PE.

Methods: A derivation cohort comprised a previously reported group of 35 consecutive patients with acute PE who were intensively studied by serial TTE at 1,2, 5 days, 2, 6, 12 and 26 weeks and RA/LA ratio related to long-term outcome. The Day 1 RA/LA ratio findings were then further related to long-term outcome in 158 patients followed for $3.6 \pm 2.3$ years.

Results: In the derivation cohort, total mortality was $28.6 \%(n=10)$ following a mean ( \pm standard deviation) follow-up of $4.3 \pm 1.9$ years. The RA/LA ratio was highly dynamic, being increased at day 1 , but normalised rapidly within 2-5 days of presentation and this was most marked amongst long-term non-survivors. A RA/LA ratio $>1.0$ on day 1 was independently associated with a three-fold increase in long-term mortality on Kaplan-Meier analysis. Pooled analysis of 158 patient indicated that age, Charlson Comorbidity Index (CCI), simplified Pulmonary Embolism Severity Score (PESI), troponin T, day 1 RA/LA Ratio and pulmonary arterial systolic pressure (PASP) were univariate predictors of long-term mortality. Multivariate analysis identified Day 1 RA/LA Ratio (HR 1.7 per 10\% increase, $p=0.002), C C l(H R 2.2$ per 1 unit increase, $p=0.004)$ and age $(H R 1.1, p=0.03)$ as the only independent predictors of long-term mortality.
\end{abstract}

Conclusion: A RA/LA Ratio $>1.0$ at presentation with acute PE was associated with a three-fold increased risk of long-term mortality. The RA/LA ratio on presentation with an acute PE is a simple, novel predictor of long-term survival.

\section{Introduction}

Acute pulmonary embolism (PE) is common and associated with an early case fatality rate of $7-11 \%[1]$, and a reported 5-year cumulative mortality rate of up to $32 \%$ [2]. Clinical scores have been developed and validated in predicting short-term prognosis following acute $\mathrm{PE}$ [3-5]. Current guidelines recommend transthoracic echocardiography (TTE) should be performed for early risk stratification following acute PE [6]. After massive $\mathrm{PE}$, right ventricular (RV) dysfunction on TTE, an

\footnotetext{
*Correspondence: leonard.kritharides@sydney.edu.au

${ }^{1}$ Concord Repatriation General Hospital and The University of Sydney, Sydney, Australia

Full list of author information is available at the end of the article
}

enlarged right atrium (RA) with reduced left atrial (LA) size on computed tomography are prominent features and indicate worse acute prognosis [6-8].

Some predictors of adverse long-term outcomes after PE have been identified. These include initial troponin elevation, baseline comorbidities as assessed by the Charlson Comorbidity Index (CCI) and ongoing functional impairment post PE [9-14]. However, relatively little is still known about the long-term outcome of patients with PE. In particular, the ability of cardiac imaging parameters obtained early during acute PE to determine long-term prognosis in patients with submassive PE who survive to hospital discharge is particularly unclear [8]. In other conditions involving right heart strain 
such as pulmonary hypertension, RV dysfunction and RA dilation both confer significantly worse long-term prognosis $[15,16]$. While quantification of RV dysfunction is still evolving, RA size, either assessed as planimetry area or as RA/LA area ratio, is robust and easily measured on TTE or on CTPA and has been shown to correlate with the severity of the pulmonary arterial obstruction in submassive PE $[17,18]$. The greater the clot burden in the pulmonary arteries, the smaller the LA area and the larger the RA area become, thus resulting in an increased RA/LA area ratio. When patients present with an acute PE to the emergency department, the RA/ LA area ratio, which can be easily measured, obtained instantaneously and is highly reproducible on echocardiography without the need for further post-processing as required by more complex strain assessment of right heart function, may be useful to the clinician. To the best of our knowledge, no study to date has examined the utility of the RA/LA area ratio, assessed early by TTE, in prognosticating the long-term mortality risk of patients post submassive PE.

The present study examined both the natural history changes to the RA/LA area ratio in patients with acute submassive $\mathrm{PE}$, and additionally investigated the longterm prognostic significance of the RA/LA area ratio in these patients. Furthermore, we sought to determine whether a specific RA/LA area ratio cutoff on echocardiography is an independent prognostic marker for longterm outcomes in patients with submassive PE.

\section{Methods}

\section{Derivation cohort}

The design of the derivation cohort study has been described previously [19]. Thirty-five patients (mean age [ \pm standard deviation] $63 \pm 18$ years) with a clinical diagnosis of PE confirmed by a high probability ventilation/ perfusion $(\mathrm{V} / \mathrm{Q})$ pulmonary scintigraphy for $\mathrm{PE}$ were prospectively recruited from 2004 in a tertiary institution. Submassive PE was defined as involvement of $30 \%$ or greater lung parenchyma and massive PE was defined as involvement of $50 \%$ or greater lung parenchyma. Biochemical profiling (cardiac troponin-T, brain natriuretic peptide $[\mathrm{BNP}], \mathrm{D}$-dimer, $\mathrm{C}$-reactive protein $[\mathrm{CRP}])$ and serial TTE assessing RV function were performed on days $1,2,5$ and weeks $2,6,12$ and 26 following admission. All patients received systemic anticoagulation for a minimum of 6 months. The overall comorbid burden and short-term mortality risk of each patient was assessed and given a Charlson Comorbidity Index (CCI) score [20] and a simplified Pulmonary Embolus Severity Index (PESI) score respectively [3]. For all survivors, final follow-up visit for a full clinical and physical examination was completed in December 2010 (mean followup $4.3 \pm 1.9$ years).

\section{Validation cohort}

A larger validation cohort was derived from a previously published series of consecutive patients from 2001-2007 with outcome status and follow-up completed in July 2008 (mean follow-up $3.8 \pm 2.6$ years) [2]. From this group of 1023 patients, 123 were identified who underwent TTE on day 1 of admission and were further studied. These patients were used as the validation cohort. The age, gender, simplified PESI score, CCI score and clinical outcomes of patients who did not undergo TTE on day 1 in the validation cohort were not significantly different from those who did not undergo a TTE on day 1 (Additional file 1: Table S1). In both derivation and validation cohorts, the outcome status of all patients was first ascertained from the state (New South Wales, Australia) death registry. Surviving patients were contacted by telephone for outcomes with 100\% complete follow-up. This study was approved by the institutional Human Research Ethics Committee.

\section{Echocardiography}

A comprehensive TTE was performed with patients in the left lateral decubitus position (Vivid5, GE Medical Systems, Milwaukee, Wisconsin). Images were acquired from standard echocardiographic views in accordance with the American Society of Echocardiography recommendations [21]. All images were stored digitally and analyzed offline using commercially available software (GE EchoPac 3.1.3, Milwaukee, Wisconsin). TTE analyses were performed in batches on anonymized images with the operator (T.C) blinded to clinical details of each study. All measurements were averaged over 3 consecutive cardiac cycles.

In the derivation cohort, the RV was assessed by conventional 2-dimensional parameters that included: the ratio of RV and left ventricular (LV) end-diastolic diameters at the basal level (tricuspid annulus); the ratio of $\mathrm{RV}: \mathrm{LV}$ end-diastolic areas; RV fractional area change [(RV end-diastolic area - RV endsystolic area)/RV enddiastolic area]; the ratio of right atrial (RA) and left atrial (LA) end-systolic areas. All areas were derived by planimetry from the apical 4-chamber view.

The pulmonary artery systolic pressure (PASP) was derived as the sum of the tricuspid regurgitant gradient obtained from continuous-wave Doppler and the right atrial pressure as estimated from the inferior vena cava [21]. The presence of McConnell's sign (distinct akinesis of the mid RV free wall with preserved apical motion), a marker of RV strain and PE severity on echocardiography, was recorded [22]. RV longitudinal function was measured by determining the tricuspid annular plane systolic excursion (TAPSE). TAPSE was determined by M-mode measurement of the displacement of the lateral tricuspid annulus during systole and diastole in the apical 4- 
chamber view [23]. Intraobserver variability was assessed by randomly selecting 10 cases and repeating all analyses on three separate occasions with the operator blinded to previous results. The variability is presented as the coefficient of variation $(\mathrm{CV})$.

In the validation cohort, the RA/LA area ratio, RV-RA pressure gradient, and the presence of RV dilatation and dysfunction were recorded [24]. In both cohorts, RV dilatation and/or dysfunction was defined as the presence of any of the following in accordance with published guidelines: ratio of RV and LV end-diastolic diameters $>0.66$; the ratio of RV:LV end-diastolic areas $>$ 0.66; RV fractional area change $<0.35$; TAPSE $<1.6 \mathrm{~cm}$ and/or presence of McConnell's sign [24].

\section{Simplified PESI and CCI scores}

The simplified PESI score is a validated index in the estimation of 30-day mortality in patients with acute PE. It incorporates the baseline demographics and comorbidities and the size of the pulmonary embolus based on hemodynamic status of the patient on admission. It is simpler to use and has similar prognostic accuracy and clinical utility as the original PESI score [3,25,26]. It comprised of six variables of equal weight (1 point per variable): age $>80$ years old, history of cancer, history of chronic cardiopulmonary disease (chronic lung disease and/or heart failure), heart rate $\geq 110$ beats per minute, systolic blood pressure $<100 \mathrm{mmHg}$ and arterial oxyhaemoglobin saturation level $<90 \%$. Patients with a simplified PESI score of $<1$ are classified as low risk and had a 30 -day mortality of $1.0 \%$ compared to $10.9 \%$ mortality amongst high risk patients (simplified PESI score $\geq 1$ ) [3].

The CCI is a summation score of the burden of comorbidities and it encompasses 17 different medical conditions including: myocardial infarction, congestive cardiac failure, peripheral vascular disease, cerebrovascular disease, dementia, chronic obstructive pulmonary disease, connective tissue disease, peptic ulcer disease, liver disease (mild vs. moderate to severe), diabetes (with or without organ damage), hemiplegia, moderate to severe renal disease, any tumor (within last 5 years), lymphoma, leukemia, metastatic solid tumor and acquired immunodeficiency syndrome [20]. It has been validated in predicting in-hospital mortality when applied to ICD-10 data [27]. A value of 0 indicates no comorbidity, while higher values represent an increasing burden of comorbid illnesses. A CCI score $\geq 3$ is associated with high risk of mortality $[28,29]$. The simplified PESI and CCI scores were both tested for their prediction of long-term mortality post PE in our two cohorts.

\section{Statistical analysis}

Data are summarized as frequencies and percentages for categorical variables. Continuous variables are presented as mean \pm standard deviation. Comparison between groups used the unpaired $t$ test for continuous variables and $\mathrm{X}^{2}$ tests or the Fisher exact test for dichotomous variables. Kaplan-Meier survival methods were used to determine long-term mortality. A single multivariate Cox proportional hazards regression analysis was performed for the entire cohort to assess for univariate predictors of mortality. Only univariate variables with $\mathrm{p}<0.05$ were included in the multivariate Cox proportional hazards regression analysis. Linear mixed modelling was used to analyse the repeated data (RA/LA Area Ratio at different pre-specified times post PE) and if significant, t-test was performed at each pre-specified time to assess for significant difference in RA/LA Area ratio between long-term survivors and non-survivors. A p value of $<0.05$ was used as a cut-off for statistical significance. Statistical analysis was performed using GraphPad Prism 5.01 (GraphPad Software, San Diego, Calif), and SPSS (Version 16.0, SPSS Inc., Chicago, Ill).

\section{Results}

Table 1 summarizes the baseline characteristics of the derivation cohort of 35 patients and the validation cohort of 123 patients. The mean extent of lung involvement assessed by pulmonary scintigraphy in the derivation cohort was $31 \pm 23 \%$ consistent with submassive PE. This was supported by the absence of in-hospital deaths during the index PE admission, with 10 (28.6\%) post discharge deaths over $4.3 \pm 1.9$ years of follow up with one death attributable to heart failure. In the validation cohort of 123 patients, there were no in-hospital deaths and 37 (30\%) post discharge deaths over $3.4 \pm 2.3$ years of follow up with six deaths attributable to heart failure. In both cohorts, the mean simplified PESI score was <1.0, $(0.9 \pm 0.9$ and $0.9 \pm 1.0$ for the derivation and validation cohorts respectively), thus implying these patients were at low risk of short-term mortality. In addition, the mean CCI score was $<3.0$ in both cohorts, suggesting a relatively low burden of comorbid disease. Thus both the derivation and validation cohorts had similar baseline and clinical characteristics as well as extent of lung involvement.

\section{Natural history of RA/LA area ratio after acute PE}

To investigate whether the initial RA/LA area ratio, or its recovery, predicted long-term outcome, we first analysed the Day $1 \mathrm{RA} / \mathrm{LA}$ area ratio as well as its subsequent change for each patient over the 6 months followup period in the derivation cohort and related this to their long-term survival (Table 2). Long-term survivors had both a lower initial and more stable RA/LA area ratio $(0.89 \pm 0.26$ and $0.83 \pm 0.12$ at day 1 and 6 months after PE). In contrast, non-survivors demonstrated acute RA dilation as evidenced by a RA/LA area ratio of $1.2 \pm$ 
Table 1 Baseline characteristics

\begin{tabular}{|c|c|c|c|}
\hline & Derivation cohort $(n=35)$ & Validation cohort $(n=123)$ & $\mathrm{p}$ value \\
\hline Follow-up (years) & $4.3 \pm 1.9$ & $3.4 \pm 2.3$ & $0.02^{*}$ \\
\hline Age, (years) & $63 \pm 18$ & $68 \pm 16$ & 0.12 \\
\hline Male, no (\%) & $18(51)$ & $60(49)$ & 0.70 \\
\hline \multicolumn{4}{|l|}{ Comorbidities, no. (\%) } \\
\hline Atrial fibrillation & $8(23)$ & $29(24)$ & 1.00 \\
\hline Diabetes & $9(26)$ & $25(20)$ & 0.49 \\
\hline Heart Failure & $2(9)$ & $21(17)$ & 0.11 \\
\hline Hypercholesterolemia & $13(37)$ & $22(18)$ & $0.02^{*}$ \\
\hline Ischaemic heart disease & $7(20)$ & $34(28)$ & 0.51 \\
\hline Malignancy & $8(23)$ & $18(15)$ & 0.13 \\
\hline Stroke & $4(11)$ & $3(2)$ & $0.04^{*}$ \\
\hline Charlson's Comorbidity Index (CCl) Score & $1.5 \pm 1.8$ & $1.6 \pm 1.9$ & 0.79 \\
\hline \multicolumn{4}{|l|}{ Clinical Outcomes } \\
\hline Day 1 PASP, (mmHg) & $33 \pm 8$ & $41 \pm 16$ & $0.006^{*}$ \\
\hline Simplified PESI score & $0.9 \pm 0.9$ & $0.9 \pm 1.0$ & 0.88 \\
\hline Recurrent PE, no (\%) & $7(20)$ & $9(7)$ & 0.05 \\
\hline Death, no (\%) & $10(29)$ & $37(30)$ & 1.00 \\
\hline
\end{tabular}

Values are presented as mean \pm SD or absolute number.

PE Pulmonary embolism, PESI Pulmonary embolism severity index, ${ }^{*} p<0.05$.

0.37 at day 1 , which decreased to $0.86 \pm 0.11$ by 6 months. However, no significant difference was present between the RA/LA area ratio of survivors and nonsurvivors when measured on day 2 after acute PE or thereafter. The apparent recovery in RA/LA area ratio on TTE between day 1 and 6 months was significantly greater in non-survivors than survivors $(-0.42 \pm 0.24$ vs.$0.01 \pm 0.12, \mathrm{p}<0.001$, respectively).

In order to identify a simple measure of RA/LA area ratio that may be of practical application, the RA/LA area ratios of the derivation cohort were analysed by tertiles. Kaplan-Meier survival analysis revealed a RA/LA area ratio $>1.03$ on day 1 admission TTE was associated with significantly worse long-term mortality (Figure 1). On this basis, an initial (day 1) RA/LA area ratio $>1.0$ was further investigated for its prognostic utility.

\section{Prognostic indicators in the combined cohort}

In the derivation cohort, day $1 \mathrm{RA} / \mathrm{LA}$ area ratio $>1.0$ was an independent predictor of long-term mortality with a hazard ratio of 5.8, adjusted for CCI score, simplified PESI score and the presence RV dilatation and dysfunction (Figure 2a). In the validation cohort, the day 1 RA/LA area ratio was significantly increased in nonsurvivors than survivors $(0.96 \pm 0.22$ vs. $0.84 \pm 0.19$, $\mathrm{p}=0.009$, respectively). A day $1 \mathrm{RA} / \mathrm{LA}$ area ratio $>1.0$ independently predicted long-term death with a hazard

Table 2 Right to left atrial area ratio and its recovery over time

\begin{tabular}{|c|c|c|c|c|}
\hline & Overall $(n=35)$ & Non-survivors $(n=10)$ & Survivors $(n=25)$ & $p$ value \\
\hline RA/LA Area Ratio D1 & $0.97 \pm 0.32$ & $1.20 \pm 0.37$ & $0.89 \pm 0.26$ & $0.01^{*}$ \\
\hline RA/LA Area Ratio D2 & $0.87 \pm 0.22$ & $0.96 \pm 0.17$ & $0.84 \pm 0.23$ & 0.23 \\
\hline RA/LA Area Ratio D5 & $0.89 \pm 0.21$ & $0.98 \pm 0.19$ & $0.84 \pm 0.21$ & 0.16 \\
\hline RA/LA Area Ratio Wk 2 & $0.83 \pm 0.24$ & $0.88 \pm 0.31$ & $0.80 \pm 0.22$ & 0.67 \\
\hline RA/LA Area Ratio Wk 6 & $0.82 \pm 0.15$ & $0.82 \pm 0.21$ & $0.82 \pm 0.13$ & 0.99 \\
\hline RA/LA Area Ratio Wk 12 & $0.90 \pm 0.20$ & $0.94 \pm 0.22$ & $0.89 \pm 0.19$ & 0.63 \\
\hline RA/LA Area Ratio Wk 26 & $0.84 \pm 0.12$ & $0.86 \pm 0.11$ & $0.83 \pm 0.12$ & 0.77 \\
\hline
\end{tabular}

Values are presented as mean \pm SD.

Linear mixed model showed significant difference in serial measurements of RA/LA area ratio over time between survivors and non-survivors, $\mathrm{p}=0.041$.

$D$ Day, Wk Week, LA Left atrium, RA Right atrium, * $\mathrm{p}<0.05$. 


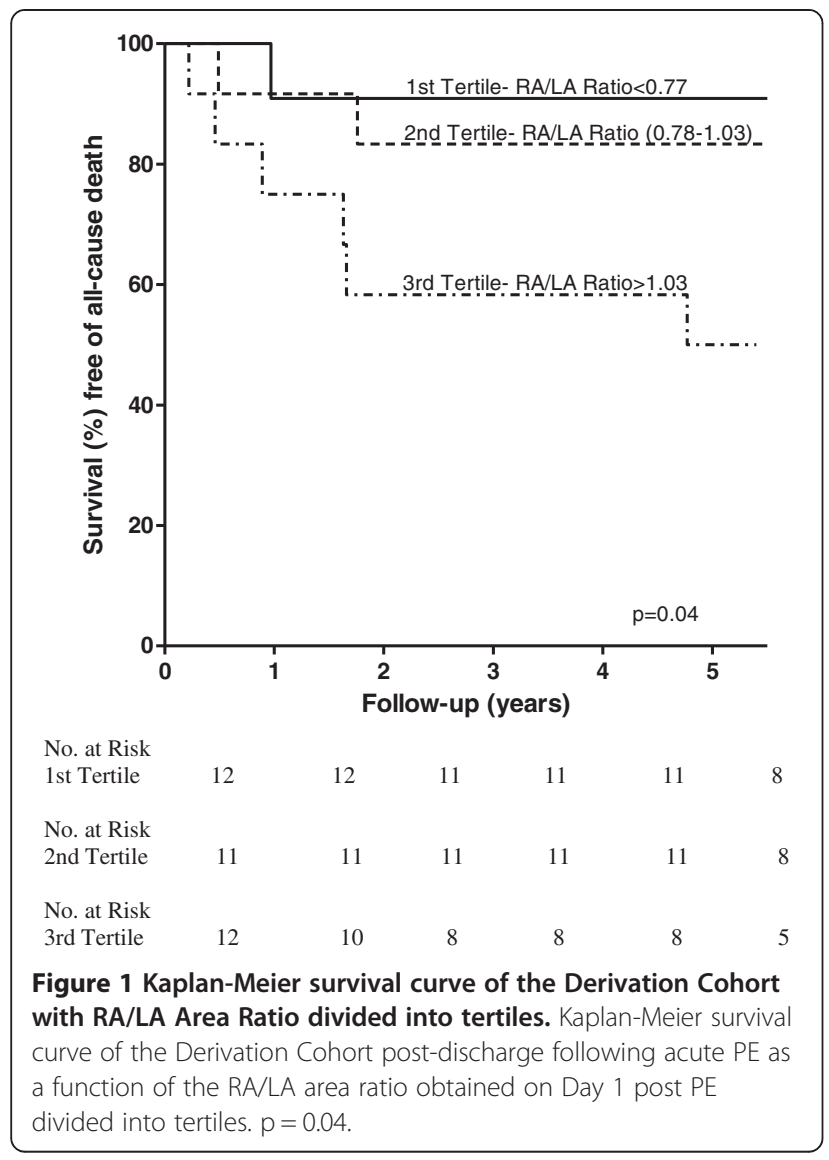

ratio of 3.0, adjusted for age, the CCI score, simplified PESI score, PASP and troponin T. (Figure 2b).

For the combined cohort, significant univariate predictors of death are shown in Table 3. These include measures of comorbidity (such as CCI score), measures of acute PE mortality risk (as measured by the simplified PESI score and Troponin T), age and measures of right heart dysfunction (including the day $1 \mathrm{RA} / \mathrm{LA}$ area ratio on day 1 and PASP).

Multivariate Cox-regression analysis using backward selection modelling identified the day $1 \mathrm{RA} / \mathrm{LA}$ area ratio (hazard ratio [HR] 1.7 per 10\% increase, 95\% confidence interval [CI] 1.2-2.4, $\mathrm{p}=0.002$ ), the CCI score (HR 2.2 per 1 unit increase, 95\% CI 1.3-3.7, $\mathrm{p}=0.004)$ and age (HR 1.1 per year increase, 95\% CI 1.0-1.2, $\mathrm{p}=0.03$ ) as independent predictors of mortality (Table 4). Intraobserver variability was less than $10 \%$ for conventional echocardiographic parameters, which is consistent with reported literature $[30,31]$. In particular, the coefficient of variation (CV) for RA:LA end-systolic area was $5 \%$.

\section{Discussion}

The ability of echocardiography to predict long-term mortality after a submassive PE is not clear. Echocardiography is recommended during the acute management
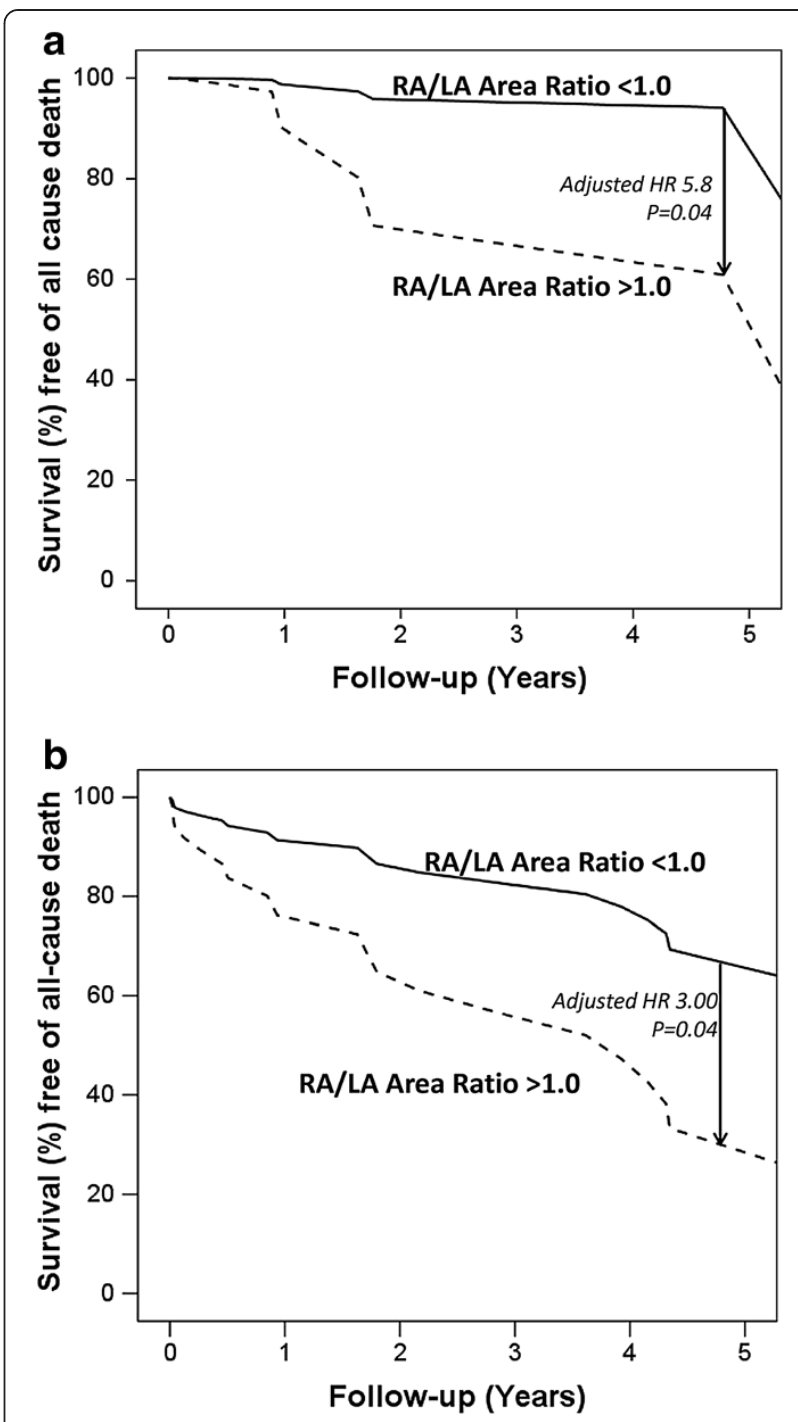

Figure 2 Kaplan-Meier survival analysis for the Derivation Cohort. a: Kaplan-Meier survival curve of the Derivation Cohort: post-discharge following acute PE utilising a cut-off of RA/LA area ratio of 1.0 on Day 1 post PE adjusted for other variables in the multivariate model. $p=0.04$. $\mathbf{b}$ : Kaplan-Meier survival curve of the Validation Cohort: post-discharge following acute PE utilising a cut-off of RA/LA area ratio of 1.0 on Day 1 post PE adjusted for other variables in the multivariate model. $p=0.04$.

of PE to assess RV dysfunction so as to further stratify non-hemodynamically compromised patients into intermediate or low-risk prognostic categories [6]. In the current study, we report for the first time that the day 1 RA/LA area ratio on echocardiography in patients with acute submassive PE predicted long-term mortality in both the derivation and validation cohorts. A day 1 RA/ LA area ratio $>1.0$ on presentation conferred at least a three-fold increase in long-term mortality. This was independent of the expected predictive value of the Charlson Comorbidity Index and simplified PESI Scores. 
Table 3 Univariate predictors of long-term mortality for the combined cohort $(\mathbf{n}=158)$

\begin{tabular}{lccc}
\hline & Hazard ratio & $\mathbf{9 5 \%} \mathbf{C l}$ & \\
\hline Baseline Characteristics & & $1.03-1.10$ \\
Age, per year increase & 1.06 & $1.4-2.2$ & $<0.001^{*}$ \\
CCl score age unadjusted, per 1 unit increase & 1.8 & $1.5-3.2$ & $<.001^{*}$ \\
Simplified PESI score, per 1 unit increase & 2.1 & $1.03-1.25$ & $<0.001^{*}$ \\
Troponin T, per 0.01 increase & 1.1 & $0.008^{*}$ \\
RV parameters on Echocardiography & & $1.1-1.5$ \\
RA/LA Area Ratio (D1), per 10\% increase & 1.3 & $0.3-1.2$ & $0.001^{*}$ \\
RV dilation and dysfunction & 0.6 & $1.01-1.07$ & 0.16 \\
PASP, per mmHg increase & 1.04 & $0.007^{*}$ \\
\hline
\end{tabular}

CCI Charlson Comorbidity Index, D1 Day 1, LA Left atrium, PASP Pulmonary artery systolic pressure, PESI Pulmonary embolus severity index, RA Right atrium, RV Right ventricle. ${ }^{*} \mathrm{p}<0.05$.

Pulmonary embolism causes an increase in RV afterload. The RA and RV are thin walled and low pressure chambers, unlike the LV, and have limited capacity to compensate to the elevated RV wall tension. This results in RV dilation and dysfunction and worsening tricuspid regurgitation.

Previous studies have shown the presence of RV dysfunction post PE, in particular an increased RV/LV ratio, by both computed tomography pulmonary angiography (CTPA) and TTE, correlated with clinical outcomes and short-term mortality [32-37]. However in other studies, no clear association could be demonstrated $[38,39]$. This discrepancy may be due to absence of an uniformly accepted standard for measuring the ventricular volumes on $\mathrm{CT}$ and the lack of electrocardiographic gating of the CTPA [40].

The RA/LA area ratio reflects the integration of multiple haemodynamic parameters including diastolic and systolic function of the RV, and filling volumes and pressures of the LA and LV. Amongst the general population without a history of pulmonary embolism, the RA/LA area ratio has been reported to be $0.81 \pm 0.15$ [41]. A raised RA/LA area ratio has previously been shown by our group to correlate with the extent of pulmonary artery occlusion after PE [18]. In acute PE, the sudden increase in RV afterload can lead to RV and RA dilation. This additionally impairs left- atrial and ventricular diastolic filling due to deviation of the inter-atrial and interventricular septae leftwards. These changes have been observed on CT scans in patients with massive PE [42,43]. A relative increase in RA and RV volumes coupled with decrease in LA and LV volumes leads to an increased RA/LA area ratio. Our results indicate that these changes are also evident in submassive PE without hemodynamic instability and are dynamic.

While it may be expected that RA/LA area ratio may relate to early outcome, the association with long-term outcome as demonstrated in the present study is novel. A particularly important finding of our study is that there is an acute recovery of the RA/LA area ratio and that this recovery does not predict a favourable longterm outcome. Therefore, TTE performed on or after day 2 of PE presentation in earlier studies are likely to underestimate the prognostic utility of the RA/LA area ratio. Pathophysiologically, our study indicates that it is the initial cardiac response to the thromboembolic insult and not the subsequent recovery that predicts long-term outcome. That the RA/LA area ratio is highly dynamic and responds acutely to volume and pressure change has been shown previously after percutaenous atrial septal defect closure. Within 24 hours of closure, Kelly et al. observed significant and immediate decrease in the RA/ LA area ratio compared with baseline TTE, despite an extended period prior with significant right sided volume overload [41]. This is due to the immediate volume and pressure unloading of the right heart, with complete normalization of the RA/LA area ratio at early followup. We postulate a similar mechanism occurs in patients

Table 4 Multivariate backward selection model for combined cohort $(n=158)$

\begin{tabular}{lccc}
\hline & Hazard ratio & 95\% Cl & p value \\
\hline RA/LA Area Ratio (D1), per 10\% increase & 1.7 & $1.2-2.4$ & $0.002^{*}$ \\
CCI score age unadjusted, per 1 unit increase & 2.2 & $1.3-3.7$ & $0.004^{*}$ \\
Age, per year increase & 1.1 & $1.0-1.2$ & $0.03^{*}$ \\
Simplified PESI score, per 1 unit increase & 2.0 & $0.9-4.4$ & 0.08 \\
\hline
\end{tabular}

CCI Charlson Comorbidity Index, D1 Day 1, LA Left atrium, PASP Pulmonary artery systolic pressure, PESI Pulmonary embolus severity index, RA Right atrium, $R V$ Right ventricle. ${ }^{*} p<0.05$. 
with acute PE where right sided volume changes occur more acutely and therefore making these changes more reversible. Following the commencement of anticoagulation therapy and the resulting reduction in thromboembolic burden in the pulmonary vasculature, there is an immediate volume and pressure unloading of the right heart with improvement in the RA/LA area ratio within 24 hours. Presumably, these patients with elevated RA/LA area ratio on Day 1 indicate a reduced cardiac-pulmonary capacity to cope with increased RV afterload, even if there is normalisation of the RA/LA area ratio over the first week of treatment independent of the initial PE size as calculated by the simplified PESI score.

The RA/LA area ratio is simple to measure and a cutoff of day $1 \mathrm{RA} / \mathrm{LA}$ area ratio $>1.0$ may be a practical guide for physicians to identify patients who are at increased risk of death in the long-term following submassive PE. In particular, we have identified 17 of the $37(46 \%)$ deaths in the validation cohort were cardiovascular or recurrent pulmonary embolus deaths which may have been preventable. These patients may benefit from long term follow-up and closer surveillance [6,7] to ensure appropriate anticoagulation therapy is provided and that there is normalisation of the RA/LA area ratio and RV function with normalisation of pulmonary haemodynamics.

The CCI is a summation score of the burden of Comorbidities [20] shown to be very useful in prognosticating the outcome of patients suffering from diseases including heart failure [44], endocarditis [45] and cancer [46]. In our current study, we confirmed the CCI is an independent predictor of long-term death following submassive PE. Among the included comorbidities in the CCI are cardiovascular diseases, chronic pulmonary diseases and malignancies. These comorbid illnesses have previously been found to be present in patients presenting with venous thromboembolism [47] and were predictors of long-term functional impairment[48] and survival [14]. We have shown for the first time that a simple and easily obtained echocardiographic parameter, the RA/LA area ratio, is an independent predictor of long-term survival following acute PE.

\section{Limitations}

This study is limited by its sample size. This may explain why cardiac troponin-T and BNP levels, which were raised amongst the non-survivors, were not independent predictors of long-term mortality following multivariate analysis in our combined cohort. We have recently demonstrated a concentration-dependent relationship between cardiac troponin-T elevation following acute PE and long-term mortality [13] and it will be important to extend the present study into larger patient cohorts to directly compare RA/LA area ratio and biochemical parameters in predicting long-term outcome.

\section{Conclusion}

In summary, patients suffering from pulmonary embolus have significantly increased long-term mortality. The early echocardiographic RA/LA area ratio is a simple, robust and easily reproducible measurement, which independently predicted long-term mortality amongst PE patients. A day $1 \mathrm{RA} / \mathrm{LA}$ area ratio $>1.0$ is independently associated with a three-fold increase in long-term mortality and this novel echocardiographic parameter may aid the long-term risk stratification following acute submassive PE.

\section{Additional file}

Additional file 1: Table S1. Comparison of baseline characteristics within the validation database between patients who did or did not undergo Day 1 TTE.

\section{Competing interests}

The authors declare that they have no competing interests.

\section{Authors' contribution}

VC, AN, TC, LZ, LK planned the study. VC and TC performed measurements and statistical analysis. All took major part in the writing and reviewing of the article. All authors have read and approved the final manuscript.

\section{Acknowledgements}

This work was supported by the Australian Postgraduate Award Scholarship (V.C.) at the University of Sydney and the Australian Government National Health and Medical Research Council Program Grant (L.K.). We thank Professor Jenny Peat (consultant statistician) for her assistance in providing statistical advice for the study.

\section{Author details}

${ }^{1}$ Concord Repatriation General Hospital and The University of Sydney, Sydney, Australia. 'Liverpool Hospital, University of New South Wales, Sydney, Australia.

Received: 8 April 2013 Accepted: 27 May 2013

Published: 31 May 2013

\section{References}

1. Stein PD, Kayali F, Olson RE: Estimated case fatality rate of pulmonary embolism, 1979 to 1998. Am J Cardiol 2004, 93:1197-1199.

2. Ng AC, Chung T, Yong AS, Wong HS, Chow V, Celermajer DS, Kritharides L: Long-term cardiovascular and noncardiovascular mortality of 1023 patients with confirmed acute pulmonary embolism. Circ Cardiovasc Qual Outcomes 2011, 4:122-128.

3. Jimenez D, Aujesky D, Moores L, Gomez V, Lobo JL, Uresandi F, Otero R, Monreal M, Muriel A, Yusen RD: Simplification of the pulmonary embolism severity index for prognostication in patients with acute symptomatic pulmonary embolism. Arch Intern Med 2010, 170:1383-1389.

4. Aujesky D, Roy PM, Le Manach CP, Verschuren F, Meyer G, Obrosky DS, Stone RA, Cornuz J, Fine MJ: Validation of a model to predict adverse outcomes in patients with pulmonary embolism. Eur Heart J 2006, 27:476-481.

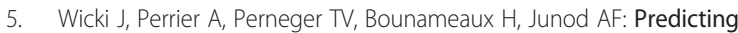
adverse outcome in patients with acute pulmonary embolism: a risk score. Thromb Haemost 2000, 84:548-552.

6. Torbicki A, Perrier A, Konstantinides S, Agnelli G, Galie N, Pruszczyk P, Bengel F, Brady AJ, Ferreira D, Janssens U, et al: Guidelines on the diagnosis and management of acute pulmonary embolism: the task force for the 
diagnosis and management of acute pulmonary embolism of the european society of cardiology (esc). Eur Heart J 2008, 29:2276-2315

7. Jaff MR, McMurtry MS, Archer SL, Cushman M, Goldenberg N, Goldhaber SZ, Jenkins JS, Kline JA, Michaels AD, Thistlethwaite $P$, et al: Management of massive and submassive pulmonary embolism, iliofemoral deep vein thrombosis, and chronic thromboembolic pulmonary hypertension: a scientific statement from the American Heart Association. Circulation 2011, 123:1788-1830.

8. Sanchez O, Trinquart L, Colombet I, Durieux P, Huisman MV, Chatellier G, Meyer G: Prognostic value of right ventricular dysfunction in patients with haemodynamically stable pulmonary embolism: a systematic review. Eur Heart J 2008, 29:1569-1577.

9. Pengo $V$, Lensing AW, Prins MH, Marchiori A, Davidson BL, Tiozzo F, Albanese P, Biasiolo A, Pegoraro C, lliceto S, Prandoni P: Incidence of chronic thromboembolic pulmonary hypertension after pulmonary embolism. N Engl J Med 2004, 350:2257-2264.

10. Becattini C, Vedovati MC, Agnelli G: Prognostic value of troponins in acute pulmonary embolism: a meta-analysis. Circulation 2007, 116:427-433.

11. Spencer FA, Gore JM, Lessard D, Douketis JD, Emery C, Goldberg RJ: Patient outcomes after deep vein thrombosis and pulmonary embolism: the Worcester Venous Thromboembolism study. Arch Intern Med 2008, 168:425-430.

12. Ng AC, Chow V, Yong AS, Chung T, Kritharides L: Prognostic impact of the charlson comorbidity index on mortality following acute pulmonary embolism. Respiration 2013, 85(5):408-16.

13. Ng AC, Yong AS, Chow V, Chung T, Freedman SB, Kritharides L: Cardiac troponin- $T$ and the prediction of acute and long-term mortality after acute pulmonary embolism. Int J Cardiol 2011, 165(1):126-33.

14. Klok FA, Zondag W, van Kralingen KW, van Dijk AP, Tamsma JT, Heyning FH, Vliegen HW, Huisman MV: Patient outcomes after acute pulmonary embolism. A pooled survival analysis of different adverse events. Am J Respir Crit Care Med 2010, 181:501-506.

15. Galie N, Hoeper MM, Humbert M, Torbicki A, Vachiery JL, Barbera JA, Beghetti M, Corris P, Gaine S, Gibbs JS, et al: Guidelines for the diagnosis and treatment of pulmonary hypertension: the Task Force for the Diagnosis and Treatment of Pulmonary Hypertension of the European Society of Cardiology (ESC) and the European Respiratory Society (ERS), endorsed by the International Society of Heart and Lung Transplantation (ISHLT). Eur Heart J 2009, 30:2493-2537.

16. McLaughlin W, Archer SL, Badesch DB, Barst RJ, Farber HW, Lindner JR, Mathier MA, McGoon MD, Park MH, Rosenson RS, et al: ACCF/AHA 2009 expert consensus document on pulmonary hypertension a report of the american college of cardiology foundation task force on expert consensus documents and the american heart association developed in collaboration with the american college of chest physicians; american thoracic society, Inc.; and the pulmonary hypertension association. J Am Coll Cardiol 2009, 53:1573-1619.

17. Aviram G, Steinvil A, Berliner S, Rosen G, Sosna J, Man A, Keren G, Rogowski $\mathrm{O}$ : The association between the embolic load and atrial size in acute pulmonary embolism. J Thromb Haemost 2011, 9:293-299.

18. Chung T, Emmett L, Khoury V, Lau GT, Elsik M, Foo F, Allman KC, Kritharides L: Atrial and ventricular echocardiographic correlates of the extent of pulmonary embolism in the elderly. J Am Soc Echocardiogr 2006, 19:347-353.

19. Chung T, Emmett L, Mansberg R, Peters M, Kritharides L: Natural history of right ventricular dysfunction after acute pulmonary embolism. J Am Soc Echocardiogr 2007, 20:885-894

20. Charlson ME, Pompei P, Ales KL, Mackenzie CR: A new method of classifying prognostic comorbidity in longitudinal studies: development and validation. J Chronic Dis 1987, 40:373-383

21. Lang RM, Bierig M, Devereux RB, Flachskampf FA, Foster E, Pellikka PA, Picard MH, Roman MJ, Seward J, Shanewise JS, et al: Recommendations for chamber quantification: a report from the american society of Echocardiography's guidelines and standards committee and the chamber quantification writing group, developed in conjunction with the european association of echocardiography, a branch of the european society of cardiology. J Am Soc Echocardiogr 2005, 18:1440-1463.

22. McConnell MV, Solomon SD, Rayan ME, Come PC, Goldhaber SZ, Lee RT: Regional right ventricular dysfunction detected by echocardiography in acute pulmonary embolism. Am J Cardiol 1996, 78:469-473.

23. Miller D, Farah MG, Liner A, Fox K, Schluchter M, Hoit BD: The relation between quantitative right ventricular ejection fraction and indices of tricuspid annular motion and myocardial performance. J Am SoC Echocardiogr 2004, 17:443-447.

24. Rudski LG, Lai WW, Afilalo J, Hua L, Handschumacher MD, Chandrasekaran K, Solomon SD, Louie EK, Schiller NB: Guidelines for the echocardiographic assessment of the right heart in adults: a report from the American Society of Echocardiography endorsed by the European Association of Echocardiography, a registered branch of the European Society of Cardiology, and the Canadian Society of Echocardiography. J Am SoC Echocardiogr 2010, 23:685-713. quiz 786-688.

25. Righini M, Roy PM, Meyer G, Verschuren F, Aujesky D, Le Gal G: The Simplified Pulmonary Embolism Severity Index (PESI): validation of a clinical prognostic model for pulmonary embolism. J Thromb Haemost 2011, 9:2115-2117

26. Venetz $C$, Jimenez $D$, Mean M, Aujesky D: A comparison of the original and simplified pulmonary embolism severity index. Thromb Haemost 2011, 106:423-428.

27. Sundararajan V, Henderson T, Perry C, Muggivan A, Quan H, Ghali WA: New ICD-10 version of the Charlson comorbidity index predicted in-hospital mortality. J Clin Epidemiol 2004, 57:1288-1294.

28. Daskivich TJ, Chamie K, Kwan L, Labo J, Palvolgyi R, Dash A, Greenfield S, Litwin MS: Overtreatment of men with low-risk prostate cancer and significant comorbidity. Cancer 2011, 117:2058-2066.

29. Rochon PA, Anderson GM, Tu JV, Clark JP, Gurwitz JH, Szalai JP, Lau P: Use of beta-blocker therapy in older patients after acute myocardial infarction in Ontario. CMAJ Canadian Medical Association Journal 1999, 161:1403-1408.

30. Kukulski T, Hubbert L, Arnold M, Wranne B, Hatle L, Sutherland GR: Normal regional right ventricular function and its change with age: a Doppler myocardial imaging study. J Am Soc Echocardiogr 2000, 13:194-204.

31. Kjaergaard J, Sogaard P, Hassager C: Right ventricular strain in pulmonary embolism by Doppler tissue echocardiography. J Am Soc Echocardiogr 2004, 17:1210-1212.

32. Quiroz R, Kucher N, Schoepf UJ, Kipfmueller F, Solomon SD, Costello P, Goldhaber SZ: Right ventricular enlargement on chest computed tomography: prognostic role in acute pulmonary embolism. Circulation 2004, 109:2401-2404.

33. Schoepf UJ, Kucher N, Kipfmueller F, Quiroz R, Costello P, Goldhaber SZ: Right ventricular enlargement on chest computed tomography: a predictor of early death in acute pulmonary embolism. Circulation 2004, 110:3276-3280.

34. van der Meer RW, Pattynama PM, van Strijen MJ, van den Berg-Huijsmans AA, Hartmann IJ, Putter $H$, de Roos A, Huisman MV: Right ventricular dysfunction and pulmonary obstruction index at helical CT: prediction of clinical outcome during 3-month follow-up in patients with acute pulmonary embolism. Radiology 2005, 235:798-803.

35. ten Wolde M, Sohne M, Quak E, Mac Gillavry MR, Buller HR: Prognostic value of echocardiographically assessed right ventricular dysfunction in patients with pulmonary embolism. Arch Intern Med 2004, 164:1685-1689.

36. Park JR, Chang SA, Jang SY, No HJ, Park SJ, Choi SH, Park SW, Kim H, Choe $\mathrm{YH}$, Lee KS, et al: Evaluation of right ventricular dysfunction and prediction of clinical outcomes in acute pulmonary embolism by chest computed tomography: comparisons with echocardiography. Int J Cardiovasc Imaging 2012, 28:979-987.

37. Kumamaru KK, Lu MT, Ghaderi Niri S, Hunsaker AR: Right ventricular enlargement in acute pulmonary embolism derived from CT pulmonary angiography. Int J Cardiovasc Imaging 2012, 28(4):965-73.

38. Araoz PA, Gotway MB, Trowbridge RL, Bailey RA, Auerbach AD, Reddy GP, Dawn SK, Webb WR, Higgins CB: Helical CT pulmonary angiography predictors of in-hospital morbidity and mortality in patients with acute pulmonary embolism. J Thorac Imaging 2003, 18:207-216.

39. Araoz PA, Gotway MB, Harrington JR, Harmsen WS, Mandrekar JN: Pulmonary embolism: prognostic CT findings. Radiology 2007, 242:889-897.

40. He H, Stein MW, Zalta B, Haramati LB: Computed tomography evaluation of right heart dysfunction in patients with acute pulmonary embolism. J Comput Assist Tomogr 2006, 30:262-266.

41. Kelly NF, Walters DL, Hourigan LA, Burstow DJ, Scalia GM: The relative atrial index (RAI)-a novel, simple, reliable, and robust transthoracic echocardiographic indicator of atrial defects. J Am Soc Echocardiogr 2010, 23:275-281

42. Hama Y, Yakushiji T, Iwasaki Y, Kaji T, Isomura N, Kusano S: Small left atrium: an adjunctive sign of hemodynamically compromised massive pulmonary embolism. Yonsei Med J 2005, 46:733-736. 
43. Ocak I, Fuhrman C: CT angiography findings of the left atrium and right ventricle in patients with massive pulmonary embolism. AJR Am J Roentgenol 2008, 191:1072-1076.

44. Clarke B, Howlett J, Sapp J, Andreou P, Parkash R: The effect of comorbidity on the competing risk of sudden and nonsudden death in an ambulatory heart failure population. Can J Cardio/ 2011, 27:254-261.

45. Sy RW, Chawantanpipat C, Richmond DR, Kritharides L: Development and validation of a time-dependent risk model for predicting mortality in infective endocarditis. Eur Heart J 2011, 32:2016-2026.

46. Albertsen PC, Moore DF, Shih W, Lin Y, Li H, Lu-Yao GL: Impact of comorbidity on survival among men with localized prostate cancer. J Clin Oncol 2011, 29:1335-1341.

47. Heit JA, Silverstein MD, Mohr DN, Petterson TM, O'Fallon WM, Melton L 3rd: Predictors of survival after deep vein thrombosis and pulmonary embolism: a population-based, cohort study. Arch Intern Med 1999, 159:445-453.

48. Klok FA, van Kralingen KW, van Dijk AP, Heyning FH, Vliegen HW, Huisman MV: Prevalence and potential determinants of exertional dyspnea after acute pulmonary embolism. Respir Med 2010, 104:1744-1749.

doi:10.1186/1476-7120-11-17

Cite this article as: Chow et al:: Right atrial to left atrial area ratio on early echocardiography predicts long-term survival after acute pulmonary embolism. Cardiovascular Ultrasound 2013 11:17.

\section{Submit your next manuscript to BioMed Central and take full advantage of:}

- Convenient online submission

- Thorough peer review

- No space constraints or color figure charges

- Immediate publication on acceptance

- Inclusion in PubMed, CAS, Scopus and Google Scholar

- Research which is freely available for redistribution 